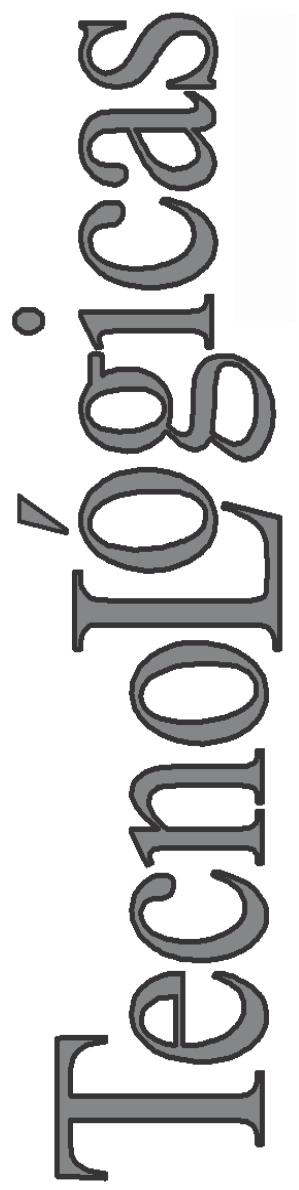

\title{
Convertidor DC/AC Multinivel de Tres Celdas: Modelado y Simulación
}

\section{Converter DC/AC Multilevel of Three Cells: Modeling and Simulation}

\author{
Julián Peláez-Restrepo ${ }^{1}$ \\ Jorge A. Herrera-Cuartas ${ }^{2}$ \\ Sergio I. Serna-Garcés ${ }^{3}$
}

1 Departamento de Electrónica y Telecomunicaciones, Instituto Tecnológico Metropolitano, Medellín-Colombia julianpelaez@itm.edu.co

2 Departamento de Electrónica y Telecomunicaciones,

Instituto Tecnológico Metropolitano, Medellín-Colombia jorgeherrera@itm.edu.co

3 Departamento de Electrónica y Telecomunicaciones, Instituto Tecnológico Metropolitano, Medellín-Colombia sergioserna@itm.edu.co 


\section{Resumen}

El presente documento presenta un convertidor DC/AC de tres celdas. Las topologías multinivel están atrayendo gran atención en la industria, ya que logran tener un rizado en las variables de estado más pequeño. Además reducen el estrés de conmutación en los dispositivos sólidos. La topología usada en este trabajo es conocida como ondulador multinivel de condensador flotante, en la cual se impone el reto de balancear la tensión entre las celdas de conmutación con ayuda de los condensadores flotantes, además de obtener una señal sinusoidal regulada. En el trabajo se expone el modelo promediado del ondulador, y los resultados obtenido mediante simulación.

\section{Palabras clave}

Convertidor DC/AC; multinivel; condensador flotante; rizado de conmutación; modelado promediado.

\section{Abstract}

This paper presents a three-cell converter DC / AC. Multilevel topologies are attracting attention in the industry, obtained as a ripple on the state variables much smaller, and reduces stress on the switching devices. The topology used in this work is known in the technical literature as floating capacitor multilevel inverter, which imposes the challenge of balancing the voltage across each cell switching using floating capacitors, besides obtaining a sinusoidal signal regulated. The paper presents the averaged model of the inverter, and results obtained through simulation.

\section{Keywords}

Converter DC/AC; multilevel; floating capacitor; switching ripple; averaging model. 


\section{INTRODUCCIÓN}

Uno de los principales obstáculos en la implementación de convertidores conmutados de potencia a alto voltaje, es el alto costo o algunas veces la inexistencia de dispositivos semiconductores que manejen altas corrientes en estado ON y simultáneamente aislé altas tensiones en estado OFF a muy altas frecuencias (Gateau, Meynard, \& Foch, 2001). Para tales limitaciones se han desarrollado varias soluciones según las necesidades de la aplicación, para altas corrientes se han propuesto soluciones como los convertidores multi-fase (Interleaved)(Mazumder, Nayfeh, \& Borojevic, 2002), mientras que para altas tensiones se proponen soluciones como los convertidores multicelulares con condensadores flotantes, que introducen una novedosa topología inspirada en los convertidores conmutados clásicos (Meynard, y otros, 2002).

La idea de los convertidores multicelulares es colocar varios dispositivos de conmutación en serie con el fin de obtener un comportamiento equivalente a un único conmutador que opera en alto voltaje a tiempos de conmutación relativamente cortos como se muestra en la Fig. 1 Sin embargo, esta forma de colocar los conmutadores no garantiza que la tensión que soporta cada conmutador esta balanceada uniformemente, ni siquiera cuando este en estado estacionario. El balance dinámico del voltaje en cada conmutador no es posible, debido a que los tiempos de encendido y de apagado de cada conmutador no son exactamente iguales.

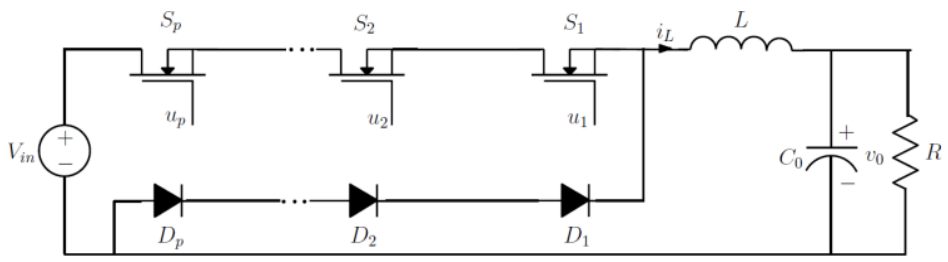

Fig. 1. Convertidor con conmutadores en serie

La topología que se propone en la Fig. 2 logra balancear el voltaje entre cada celda de conmutación sin aumentar el orden del sistema, pero es demasiado costosa ya que implica que además de la fuente de alimentación, debemos disponer de $p-1$ fuentes de tensión adicionales. 
La solución que se ha propuesto para este problema, es colocar en lugar de fuentes de alimentación de valor $r V i n / p$ donde $r=1, \cdots, p-1$ colocar un condensador entre cada celda, y ejercer una acción de control que asegure que el condensador este siempre a un valor de tensión $r V i n / p$ aumentando así el orden del sistema ya que cada condensador es una nueva variable de estado a controlar, además de la corriente de inductor y el voltaje del condensador de salida.

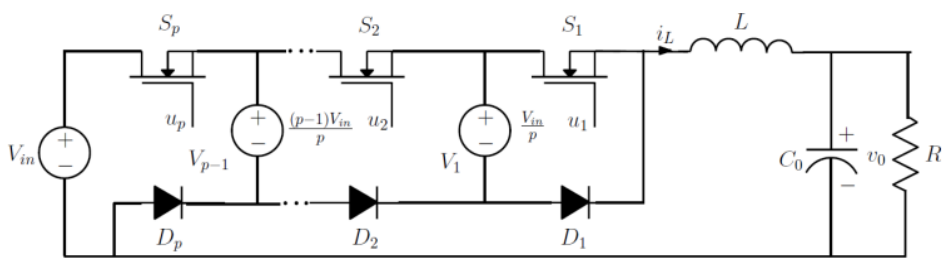

Fig. 2. Convertidor con conmutadores en serie, balanceado con fuentes de tensión

Así, la idea básica de los convertidores multinivel es un arreglo de dispositivos de conmutación con un condensador flotante entre cada celda de conmutación (Lienhardt, Gateau, \& Meynard, 2005), compuesta por el par MOSFET-diodo o conmutador controlado y complementario. En la Fig. 3 vemos un convertidor multinivel de $p$ celdas.

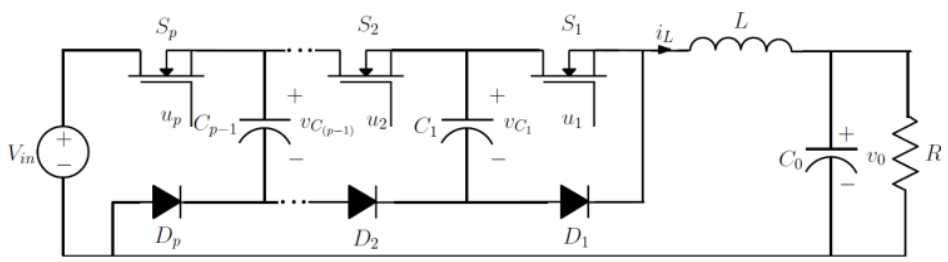

Fig. 3. Convertidor con conmutadores multinivel de p celdas de conmutación

\section{METODOLOGía}

En un ondulador debido a la naturaleza bidireccional de la corriente, no podremos utilizar diodos ya que estos se polarizan en una única dirección de la corriente, por lo tanto para una topología de un ondulador multinivel de tres celdas de conmutación, donde 
se han reemplazado los diodos por MOSFET, la podemos observar en la Fig. 4.

De acuerdo con (Peláez-Restrepo, 2011), en el cual se establece que las señales de control deben ser iguales en estado estacionario pero desfasadas $2 \pi / p$ entre ellas y diferentes en los transitorios, además que las funciones de transferencia de los capacitores flotantes son integradores puros por lo se pueden controlar con una simple ganancia proporcional (Wilkinson, Mouton, \& Meynard, 2003), pero el lazo de control del voltaje de salida tiende a ser inestable y a tener un error de estado estacionario diferente de cero.

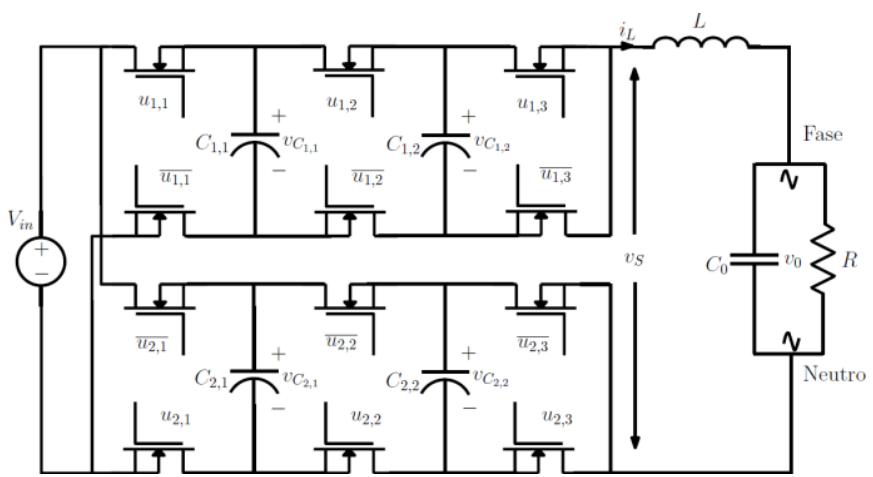

Fig. 4. Ondulador multinivel de tres celdas de conmutación de puente $\mathrm{H}$ completo

La estrategia de control propuesta es mostrada en la Fig. 5, donde se puede observar claramente las señales de los ciclos útiles de cada uno de los conmutadores controlados, los no controlados da por hecho que sus señales son complementarias
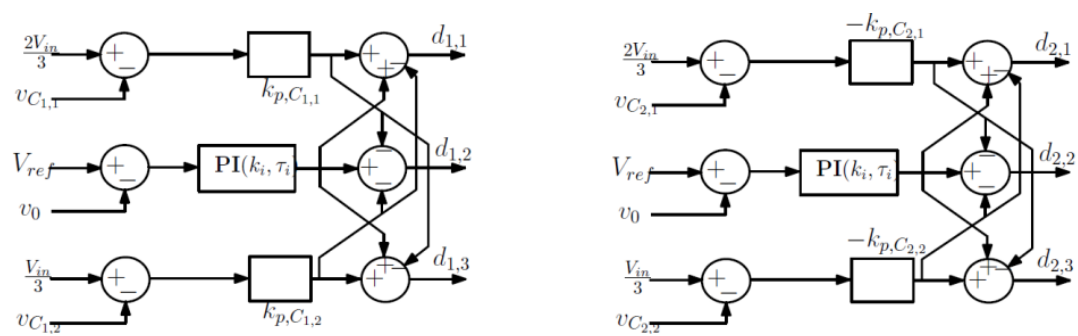

Fig. 5. Esquema de control del ondulador de tres celdas 


\subsection{Modelado del Ondulador Multinivel de Tres Celdas de Conmutación}

El inversor de tres celdas expuesto en este documento, presenta una serie de posibles estados de conmutación como se ve en la Tabla 1, el modelo promediado se puede hallar sumando cada uno de los espacios de estados del circuito equivalente, multiplicado por la configuración correspondiente (ver (1)). Y finalmente despreciando los términos que no sean combinación lineal entre las variables de estado y las señales de conmutación (Sun \& Grotstollen, 1997).

Tabla 1. Configuraciones de los diferentes estados del ondulador multicelular

\begin{tabular}{cccccccc}
$U_{13}$ & $U_{13}$ & $U_{13}$ & $U_{13}$ & $U_{13}$ & $U_{13}$ & Secuencia & Configuración \\
\hline 0 & 0 & 0 & 1 & 1 & 1 & $\overline{U_{13} U_{12} U_{11}}$ & $C_{1}$ \\
0 & 0 & 1 & 1 & 1 & 1 & $\overline{U_{13} U_{12}} U_{11}$ & $C_{2}$ \\
0 & 1 & 0 & 1 & 1 & 1 & $\overline{U_{13}} U_{12} \overline{U_{11}}$ & $C_{3}$ \\
0 & 1 & 1 & 1 & 1 & 1 & $\overline{U_{13}} U_{12} U_{11}$ & $C_{4}$ \\
1 & 0 & 0 & 1 & 1 & 1 & $U_{13} \overline{U_{12}} U_{11}$ & $C_{5}$ \\
1 & 0 & 1 & 1 & 1 & 1 & $U_{13} \overline{U_{12}} U_{11}$ & $C_{6}$ \\
1 & 1 & 0 & 1 & 1 & 1 & $U_{13} U_{12} \overline{U_{11}}$ & $C_{7}$ \\
1 & 1 & 1 & 1 & 1 & 1 & $U_{13} U_{12} U_{11}$ & $C_{8}$ \\
0 & 0 & 0 & 0 & 0 & 0 & $\overline{U_{23} U_{22}} \bar{U}_{21}$ & $C_{9}$ \\
0 & 0 & 0 & 0 & 0 & 1 & $\overline{U_{23} U_{22}} U_{21}$ & $C_{10}$ \\
0 & 0 & 0 & 0 & 1 & 0 & $\overline{U_{23}} U_{22} \overline{U_{21}}$ & $C_{11}$ \\
0 & 0 & 0 & 0 & 1 & 1 & $\overline{U_{23}} U_{22} U_{21}$ & $C_{12}$ \\
0 & 0 & 0 & 1 & 0 & 0 & $U_{23} \overline{U_{22}} \overline{U_{21}}$ & $C_{13}$ \\
0 & 0 & 0 & 1 & 0 & 1 & $U_{23} \overline{U_{22}} U_{21}$ & $C_{14}$ \\
0 & 0 & 0 & 1 & 1 & 0 & $U_{23} U_{22} \overline{U_{21}}$ & $C_{15}$ \\
0 & 0 & 0 & 1 & 1 & 1 & $U_{23} U_{22} U_{21}$ & $C_{16}$ \\
\hline
\end{tabular}

$\dot{x}=\sum_{k=1}^{16}\left(A_{k} x+B_{k}\right) C_{k}$ 
En (2), (3), (4) vemos el modelo promediado del convertidor, en lazo cerrado, con un control PI.

$\dot{x}=A_{\text {avg }} x+B_{\text {avg }}$

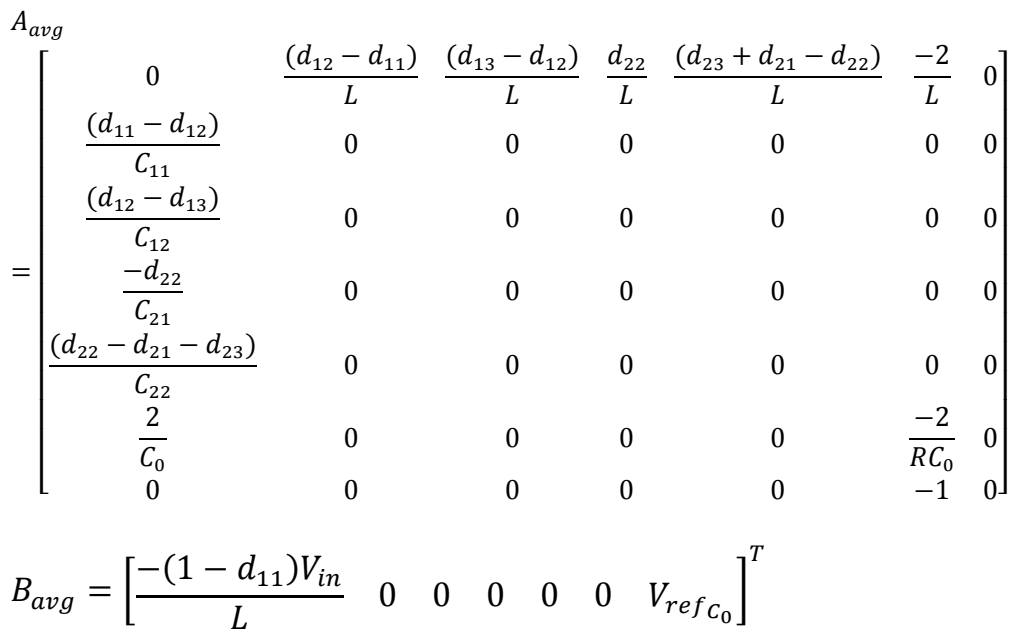

Finalmente las señales de control del convertidor son (5).

$$
\begin{aligned}
& d_{11}=k_{p}\left(V_{\text {ref }_{C_{11}}}-x_{2}\right)+\frac{k_{i}}{\tau_{i}} x_{4}+k_{i}\left(V_{\text {ref }_{C_{0}}}-x_{6}\right)-k_{p}\left(V_{\text {ref }_{C_{12}}}-x_{3}\right) \\
& d_{12}=-k_{p}\left(V_{\text {ref }_{C_{11}}}-x_{2}\right)+\frac{k_{i}}{\tau_{i}} x_{4}+k_{i}\left(V_{\text {ref }_{C_{0}}}-x_{6}\right)+k_{p}\left(V_{\text {ref }_{C_{12}}}-x_{3}\right) \\
& d_{13}=-k_{p}\left(V_{\text {ref }_{C_{11}}}-x_{2}\right)+\frac{k_{i}}{\tau_{i}} x_{4}+k_{i}\left(V_{\text {ref }_{C_{0}}}-x_{6}\right)-k_{p}\left(V_{\text {ref }_{C_{12}}}-x_{3}\right) \\
& d_{21}=-k_{p}\left(V_{\text {ref }_{C_{21}}}-x_{4}\right)+\frac{k_{i}}{\tau_{i}} x_{4}+k_{i}\left(V_{\text {ref }_{C_{0}}}-x_{6}\right)+k_{p}\left(V_{\text {ref }_{C_{22}}}-x_{5}\right) \\
& d_{22}=k_{p}\left(V_{\text {ref }_{C_{21}}}-x_{4}\right)+\frac{k_{i}}{\tau_{i}} x_{4}+k_{i}\left(V_{\text {ref }_{C_{0}}}-x_{6}\right)-k_{p}\left(V_{\text {ref }_{C_{22}}}-x_{5}\right) \\
& d_{23}=k_{p}\left(V_{\text {ref }_{C_{21}}}-x_{4}\right)+\frac{k_{i}}{\tau_{i}} x_{4}+k_{i}\left(V_{\text {ref }_{C_{0}}}-x_{6}\right)+k_{p}\left(V_{\text {ref }_{C_{22}}}-x_{5}\right)
\end{aligned}
$$




\section{RESULTADOS}

Finalmente se pueden obtener las simulaciones del convertidor en PSIM, con los valores de parámetros dados en la Tabla 2.

Tabla 2. Valores de los parámetros

\begin{tabular}{ccc}
\hline Parametro & Valor & Unidades \\
\hline$C_{11}, C_{21}$ & 20 & $\mu F$ \\
$C_{12}, C_{22}$ & 10 & $\mu F$ \\
$C_{0}$ & 10 & $\mu F$ \\
$L$ & 0.1 & $m H$ \\
$R$ & 10 & $\Omega$ \\
$V_{\text {in }}$ & 120 & $V$ \\
$V_{\text {ref }_{C_{11}}} V_{\text {ref }} C_{21}$ & $\frac{2}{3} V_{\text {in }}$ & $V$ \\
$V_{\text {ref }}{ }_{C_{12}}, V_{\text {ref }} C_{22}$ & $\frac{1}{3} V_{\text {in }}$ & $V$ \\
$V_{\text {ref }} C_{C_{0} 0}$ & $110 \sin (2 \pi f)$ & $V$ \\
$f$ & 60 & $\mathrm{~Hz}$ \\
$f_{s}$ & 60 & $\mathrm{kHz}$ \\
$k_{p}$ & 0.05 & \\
$k_{i}$ & 0.25 & \\
$\tau_{i}$ & $1 E-4$ & $\mathrm{Seg}$ \\
\hline
\end{tabular}

En la Fig. 6 podemos ver la forma de onda del voltaje de salida, después del filtro LC, el cual es prácticamente sinusoidal, además podemos observar las señales de control de cada uno de los conmutadores y vemos que el semi-ciclo positivo solo actúan las señales $u_{1, x}$, mientras que en el semi-ciclo negativo actúan las señales $u_{2, x}$, lo cual se explica claramente por la topología en forma de puente $\mathrm{H}$.

Además en la Fig. 7 podemos observar claramente el balance natural (Wilkinson, Mouton, \& Meynard, 2003) de las tensiones en los condensadores flotantes, lo cual nos asegura un correcto balance entre las tensiones que soportan cada uno de los dispositivos de conmutación, por lo que un inversor de alto voltaje con ayuda de 
esta topología se podría implementar con conmutadores de propósito general.
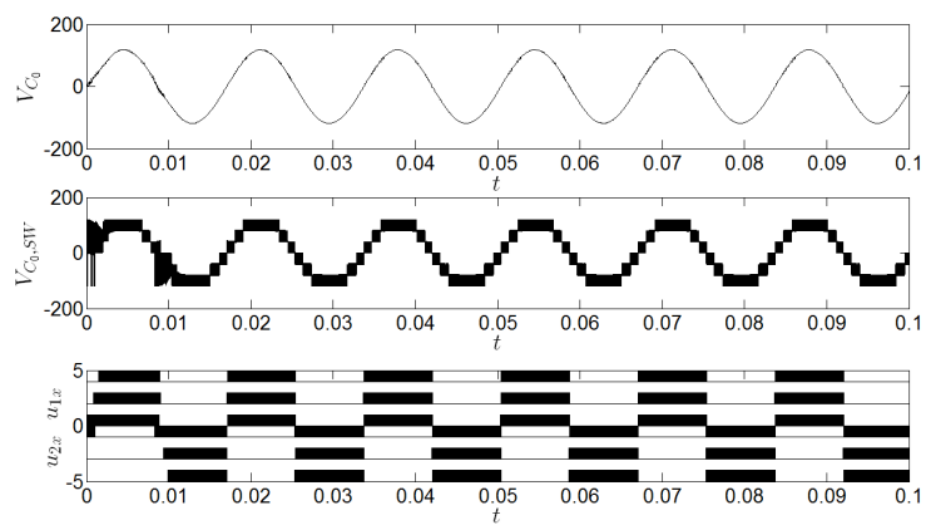

Fig. 6. Señal sinodal regulada y las señales de control de cada conmutador

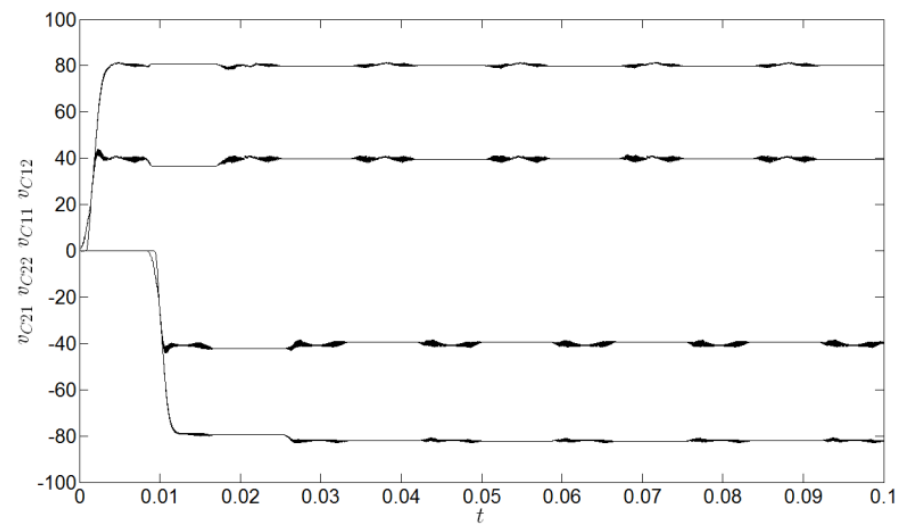

Fig. 7. Balance natural del voltaje de los condensadores flotantes

\section{CONCLUSIONES}

Los convertidores multicelulares, son apropiados para potencias medias y altas, donde no es posible conseguir comerciablemente dispositivos que aíslen altas tensiones a relativamente altas frecuencias. Además mejoran el rendimiento del sistema al gene- 
rar menor estrés en los conmutadores ( $\mathrm{Su}, 2005)$, aumentar la frecuencia de conmutación aparente y por lo tanto los valores del rizado de las variables de estado son mucho más pequeños. Este tipo de onduladores es ideal para el manejo de catenarias en un tranvía eléctrico, como drivers de motores de inducción de gran tamaño (Meynard, y otros, 2002), y en un tipo de FACTS utilizada en líneas de distribución llamados D-STATCOM, los cuales prometen mucho en las redes SMART GRID para mantener una buena calidad energética.

\section{REFERENCIAS}

Gateau, G., Meynard, T., \& Foch, H. (2001). Stacked Multicell Converter (SMC): Properties and design. IEEE Transactions on Power Electronics, 8(1), 1583-1588.

Lienhardt, A., Gateau, G., \& Meynard, T. (2005). Stacked Multicell Converter (SMC): Estimation of Flying Capacitor Voltages. 11 European Conference on Power Electronics and Applications EPE'05, 1-10.

Mazumder, S. K., Nayfeh, A. H., \& Borojevic, D. (2002). Robust Control of Parallel DC-DC Buck Converters by Combining Integral-VariableStructure and Multiple-Sliding-Surface Control Schemes. IEEE TRANSACTIONS ON POWER ELECTRONICS, 17(3), 428-437.

Meynard, T., Foch, H., Thomas, P., Courault, J., Jacob, R., \& Nahrstaedt, M. (2002). Multicell converters: basic concepts and industry applications. IEEE Transactions on Industrial Electronics, 49(5), 955-964.

Peláez-Restrepo, J. (2011). Contribución al estudio de la dinámica y control de un convertidor buck de dos celdas de conmutación. (DEEEA, Ed.) Tesis Doctoral, 229.

Su, G.-J. (2005). Multilevel DC-Link Inverter. IEEE TRANSACTIONS ON INDUSTRY APPLICATIONS, 41(3), 848-854.

Sun, J., \& Grotstollen, H. (1997). Symbolic Analysis Methods for Averaged Modeling of Switching Power Converters. IEEE TRANSACTIONS ON POWER ELECTRONICS, 12(3), 537-546.

Wilkinson, R., Mouton, H. d., \& Meynard, T. (2003). Natural Balance of Multicell Converters. IEEE Transactions on Industrial Electronics, 1307-1312. 